\title{
Shoulder Celbow
}

\section{Thanks to Reviewers}

Shoulder \& Elbow

2018, Vol. I0(2) 144-145

(C) The Author(s) 2018

Reprints and permissions: sagepub.co.uk/journalsPermissions.nav DOI: I0.I I77/I7585732।8758850

journals.sagepub.com/home/sel

SAGE

The journal sincerely thanks the following individuals who reviewed one or more manuscripts during 2017:

\begin{tabular}{ll} 
Adolfsson, Lars & Cuff, Andrew \\
Ahrens, Philip & Deshmukh, Subodh \\
Ali, Amjid & Dey, Roopam \\
Aly, Amr & Dhawan, Rohit \\
Amirfeyz, Rouin & Donaldson, Oliver \\
Ankarath, Sudhi & Douglas, Tania \\
Anley, Cameron & Drew, Steve \\
Antuna, Samuel & Duke, Phil \\
Armstrong, Alison & Evans, Mathew \\
Bale, R & Eygendaal, Denise \\
Baratz, Mark & Falworth, Mark \\
Barco, Raul & Farrar, Mark \\
Bateman, Marcus & Fehringer, Edward \\
Bell, Simon & Frostick, Simon \\
Berber, Onur & Gandhi, Maulik \\
Bidwai, Amit & Gardner, Andrew \\
Birch, Ann & Gibson, Jo \\
Blacknall, James & Goodchild, Lorna \\
Blackwell, John & Gooding, Benjamin \\
Boardman, David & Gregory, Jonathon \\
Booker, Simon & Guisasola, Inigo \\
Boyle, Simon & Guyver, Paul \\
Brooksbank, Andrew & Hackney, Roger \\
Brownlow, Harry & Harrington, Paul \\
Brownson, Peter & Hay, Stuart \\
Bull, Anthony & Hearnden, Anthony \\
Burton, David & Hewitt, Catherine \\
Candal-Couto, Jaime & Higgs, Deborah \\
Carr, Ashley & Hillen, Robert Jan \\
Chambler, Andrew & Holland, Philip \\
Charalambous, Charalambos & Horsley, Ian \\
Chua, Soong & Hoy, Gregory \\
Cicak, Nikola & Hughes, Peter \\
Clark, David & Ibrahim, Edward \\
Clasper, Jon & Ingoe, Helen \\
Clitherow, Harry & Iossifidis, Anestis \\
Cloke, David & Jaggi, Anju \\
Colaço, Henry & Jenkins, Paul \\
Cole, Andrew & Jepson, Alistair \\
Cole, Andy & Jones, Val \\
Colegate-Stone, Toby & Jordaan, Pieter \\
Connor, Clare & Kaas, Laurens \\
Cool, Paul & Kamineni, Srinath \\
Cowling, Paul & \\
Crowther, Mark & \\
& Kenty \\
\hline
\end{tabular}

Adolfsson, Lars

Ahrens, Philip

Ali, Amjid

Aly, Amr

Amirfeyz, Rouin

Ankarath, Sudhi

Anley, Cameron

Antuna, Samue

Bale, $\mathrm{R}$

Mark

Bell, Simon

Berber, Onur

Bidwai, Ami

Birch, Ann

Blacknall, James

Blackwell, John

Boardman, David

Booker, Simon

Boyle, Simon

Brooksbank, Andrew

Burton, David

Candal-Couto, Jaime

Carr, Ashley

Chambler, Andrew

Charalambous, Charalambos

Chua, Soong

Cicak, Nikola

Clark, David

Clasper, Jon

Clitherow, Harry

Cloke, David

Colaço, Henry

Colegate-Stone, Toby

Connor, Clare

Cowling, Paul

Crowther, Mark

\author{
Cuff, Andrew \\ Dey, Roopam \\ Dhawan, Rohit \\ Donaldson, Oliver \\ Duke, Phil \\ Evans, Mathew \\ ygendaal, Denise \\ Farrar, \\ Fehringer, Edward \\ Gandhi, Maulik \\ Gardner, Andrew \\ Gibson, Jo \\ Goodchild, Lorna \\ Gooding, Benjamin \\ Gregory, Jonathon \\ Guisasola, Inigo \\ Guyver, Paul \\ ackney, Roger \\ Hay, Stuart \\ Hearnden, Anthony \\ Higgs, Deborah \\ Hillen, Robert Jan \\ Holland, Philip \\ Horsley, Ian \\ Hoy, Gregory \\ Hughes, Peter \\ brahim, Edward \\ Iossifidis, Anestis \\ aggi, Anju \\ kins, Paul \\ n, Alistair \\ Jordaan, Pieter \\ Las, Laurens \\ Kent, Matthew
}

King, Graham

Kumar, Kapil

Kumar, Shyam

Kurer, Michael

Lambert, Simon

Lawrence, Tom

Le Leu, Andre

Levy, Ofer

Limb, David

Littlewood, Chris

Lozano-Calderon, Santiago

Mackenzie, Tanya

Major, Daniel

Matthews, Stuart

Mazuquin, Bruno

Mcbirnie, Julie

McVie, James

Michener, Lori

Miller, David

Mishra, Allan

Moaveni, Ash

Modi, Amit

Modi, Chetan

Monga, Puneet

Moulton, Lawrence

Mullett, Hannan

Nanda, Rajesh

Negru, Marius

Nuttall, David

Olsen, Bo

Ostendorf, Marieke

Page, Richard

Pandey, Radhakant

Parmar, Rish

Patel, Minoo

Patel, Vipul

Payne, Carol

Peach, Chris

Pennington, Neil

Phadnis, Joideep

Poon, Peter

Pratt, Roland

Pritchard, Mark

Proper, Stewart

Pulavarti, Ramnadh 
Rashid, Abbas

Ravenscroft, Matt

Redfern, Tom

Richards, Andrew

Roche, Stephen

Rotstein, Andrew

Rouleau, Dominique

Rumian, Adam

Salomonsson, Bjorn

Sarda, Praveen

Scheibel, Markus

Scott, Martin

Shahane, Shantanu

Sheps, David

Sibly, Frank

Singh, Harvinder pal

Sinopidis, Christos

Skare, Øystein

Smith, Christopher
Smith, Geoffrey

Smith, Matthew

Spurrier, Edward

Struyf, Filip

Talbot, Joseph

Tambe, Amol

Temperley, David

Tennent, Duncan

The, Bertram

Theivendran, Kanthan

Thomas, Michael

Thomas, William

Thyagarajan, David

Trail, Ian

Tytherleigh-Strong, Graham van Deurzen, Derek

van Raay, Jos

van Riet, Roger

Van Tongel, Alexander
Vannet, Nicola

Venkateswaran, Balachandran

Visser, Cornelis

Vollans, Sam

Vrettos, Basil

Vuillermin, Carley

Wallace, Andrew

Walton, Julia

Walton, Michael

Watson, Lyn

Watts, Adam

Webb, Mark

Willems, Jaap

Williams, John

Woods, David

Wright, Warwick

Wykes, Philip

Yewlett, Alun 